\section{GENRE}

en séries

\section{Genre en séries}

Cinéma, télévision, médias

9 | 2019

Cultures juvéniles de l'amour

\title{
Autonomie, sensibilité et dévouement : genre et appropriations culturelles des histoires d'amour à l'âge du lycée
}

\section{Viviane Albenga}

\section{(2) OpenEdition \\ Journals}

Édition électronique

URL : http://journals.openedition.org/ges/351

DOI : $10.4000 /$ ges.351

ISSN : 2437-6563

Éditeur

Presses universitaires de Bordeaux

\section{Référence électronique}

Viviane Albenga, «Autonomie, sensibilité et dévouement : genre et appropriations culturelles des histoires d'amour à l'âge du lycée », Genre en séries [En ligne], 9 | 2019, mis en ligne le 01 mai 2019, consulté le 18 février 2021. URL : http://journals.openedition.org/ges/351 ; DOI : https://doi.org/ $10.4000 /$ ges.351

Ce document a été généré automatiquement le 18 février 2021.

\section{(c) (i) ()}

La revue Genre en séries est mise à disposition selon les termes de la Licence Creative Commons Attribution - Pas d'Utilisation Commerciale - Pas de Modification 4.0 International. 


\title{
Autonomie, sensibilité et dévouement : genre et appropriations culturelles des histoires d'amour à l'âge du lycée
}

\author{
Viviane Albenga
}

«Au départ c'est un voyou puis il évolue parce
qu'il est amoureux d'elle et il fait tout pour elle
alors qu'au début il était pas très sympa avec
elle »; « C'est une fille qui avait 18 ans, elle a
jamais eu de copain et la première fois qu'elle est
tombée amoureuse c'est sa première fois, et il la
trompe »; " il n' y a pas beaucoup d'histoire
d'amour qui se passent bien, celles qu'on retient
c'est pas celles qui se passent le mieux »; « Léo il
est entièrement dévoué à sa copine, il ne fait pas
semblant il est amoureux et il fait tout pour elle ».

Ces citations de lycéen.ne.s que nous avons recueillies condensent des représentations de l'amour issues de livres, de séries et de films. Si l'apprentissage amoureux par les livres (Baudelot, Cartier et Détrez, 1999) ou par les séries télévisées comme Hélène et les garçons (Pasquier, 1999) a bien été attesté, qu'en est-il aujourd'hui des effets de biens culturels qui racontent des histoires d'amour à l'âge du lycée ? En effet, l'âge moyen au premier rapport hétérosexuel en France demeurant 17 ans (Bajos et Bozon, 2008), le moment du lycée permet de saisir des parcours différenciés entre ceux et celles qui ont déjà construit un parcours amoureux et sexuel, ceux et celles qui le débutent, ou encore les adolescent.e.s qui tout en ayant déjà fait l'expérience d'émotions amoureuses, n'ont pas encore vécu de relations. Si « l'amour » comme "pratique sociale» (Bozon, 2016) a une définition susceptible de varier selon les âges, les classes sociales et le genre (Clair, 2007, 2011), dans le cas de lycéens et lycéennes ces lignes sont brouillées par d'autres 
différenciations, comme celles des trajectoires amoureuses. À ce titre, les pratiques culturelles nous renseignent sur les "scripts" amoureux des enquêté.e.s à ce stade de leur trajectoire amoureuse. Isabelle Clair invite les sociologues à étendre le concept de script sexuel de John Gagnon (2008), qui a «d'abord visé à penser la façon dont les individus entrent en relation sexuelle les uns avec les autres", à d'autres types de relations sociales, ici les relations amoureuses. Comme elle le souligne, « la théorie des scripts sexuels est utile [...] parce que, forgée pour comprendre la sexualité humaine, elle peut être transposée à toute relation qui suit un agencement de séquences à la fois préétabli et tributaire des aléas des interactions sociales et des histoires individuelles. » (Clair, 2016 : 11). Les scripts gagnent à être analysés au prisme du schéma narratif de la romance, dont Janice Radway (1984) a mis au jour le caractère genré et patriarcal.

2 À partir d'une enquête réalisée auprès de 77 lycéens et lycéennes dans le cadre d'un projet collectif financé par le Ministère de la Culture, je vais montrer que les appropriations - i.e. le sens donné aux œuvres ainsi que les sociabilités, échanges et discussions autour de ces œuvres (Chartier, 1987) - des films, livres, séries et chansons reconduisent et reconfigurent les rapports de genre qui traversent les relations amoureuses. Deux dimensions des rapports de genre dans les romances peuvent être soulignées : la première a trait aux positions asymétriques occupées par les hommes et les femmes dans les relations amoureuses, qui se traduit dans le schéma narratif de la romance par l'histoire d'une femme qui transforme un homme distant, peu sensible voire brutal et violent en mari affectueux et attentionné (Radway, 1984); la seconde à la binarité de genre sous-tendue par le modèle implicite de deux sexes complémentaires dans la relation amoureuse hétérosexuelle. En outre, les rapports de genre dans les romances reflètent et exagèrent une asymétrie réelle de l'investissement émotionnel entre hommes et femmes: c'est ce qu'Arlie Hoshchild (2017) identifie comme un "travail émotionnel ", lequel détient une valeur d'échange dans la sphère professionnelle contre une valeur d'usage dans la sphère privée. Hoshchild démontre que le ressenti des émotions et leur compréhension implique un travail profond de transformation de soi. Le modèle de la romance tel qu'analysé par Radway traduit narrativement le travail effectué par les femmes pour changer les hommes et les amener au mariage.

3 Si Janice Radway s'est intéressée à la réception de la romance par ses lectrices, j'analyserai ici la réception et la réappropriation de ce schéma sur un corpus plus large par des lycéens et lycéennes. Je m'inspirerai des propositions de Hans R. Jauss (1978) qui érigent l'art en vecteur de changement social par ce qu'il suscite comme émotions qui viennent bouleverser les horizons d'attente. Les analyses de Jauss sur la dimension émotionnelle des identifications aux personnages ont déjà été rendues opératoires en sociologie de la lecture et du genre (Albenga, 2011 ; Détrez, 2011). Le grand intérêt des écrits de Jauss réside dans le fait d'avoir défini, en s'appuyant sur la Poétique d'Aristote, une identification non seulement empathique à des personnages «qui nous ressemblent", mais également une identification admirative "envers des héros meilleurs que nous ", et cathartique envers des personnages pris dans des situations extrêmes, comiques ou tragiques. L'apprentissage des relations amoureuses accompagne même des identifications cathartiques à des histoires difficiles voire sordides (Baudelot, Cartier et Détrez, 1999).

Dans une première partie, je ciblerai l'analyse sur les stéréotypes de genre liés au script narratif de la romance. Cette analyse s'appuiera sur les discours tenus sur l'amour par 
les enquêté.e.s à partir des œuvres et des personnages féminins et masculins considérés comme marquants, qu'ils s'inscrivent dans des scripts amoureux ou non. Je mettrai en évidence la reconduction des stéréotypes relatifs au rôle négatif des hommes dans la romance, qui tient de l'identification cathartique, puis la recherche de contrestéréotypes de la part de lycéens et lycéennes qui consomment des biens culturels en quantité et manifestent une identification admirative envers des personnages féminins forts et indépendants, ainsi que des personnages masculins sensibles. Puis dans une seconde partie, je questionnerai la portée égalitaire du script amoureux alternatif de dévouement amoureux réciproque revendiqué par les filles et les garçons. Je montrerai d'une part qu'il s'accommode de transgressions à la norme de l'hétérosexualité sans remettre en cause le duo conjugal ; et d'autre part que sa portée alternative est limitée par la persistance d'une prise en charge plus grande par les filles du travail émotionnel.

\section{Encadré méthodologique}

Deux phases d'enquête ont permis de réunir environ quatre-vingts entretiens réalisés auprès de lycéen.ne.s de Première et Terminale quasiment exclusivement en filière générale (seulement 3 sont en filière technologique et 5 en bac professionnel). J'ai réalisé dix-neuf entretiens dans deux lycées bordelais, puis j'ai dirigé deux classes d'étudiant.e.s en 2e année de DUT Métiers du Livre (une classe de DUT Bibliothèques-Médiathèques-Patrimoine et une classe de DUT éditionLibrairie) qui ont récolté cinquante-huit entretiens analysables. Les deux classes ont élaboré des hypothèses et une grille d'entretien en commun. Ils ont également élaboré une liste d'œuvres pour qu'elle soit présentée aux enquêté.e.s qui pouvaient sélectionner des œuvres qu'ils avaient aimées et en parler. Entre la première et la seconde phase d'enquête, des relances ont été ajoutées à la grille d'entretien pour connaître les personnages masculins et féminins considérés comme marquants, qu'ils soient ou non associés à la thématique de l'amour.

La population enquêtée est composée de davantage de filles (47 sur 77 entretiens si l'on ajoute ceux que j'ai réalisés et ceux des étudiant.e.s), et très majoritairement située dans l'espace des classes moyennes. Ces biais reflètent les caractéristiques sociales des étudiant.e.s de DUT qui ont contacté les élèves interviewé.e.s par leur réseau d'interconnaissance. S'ils empêchent d'embrasser tout le spectre des différences de classe, ils permettent en revanche de mettre en évidence les effets de la forte consommation de biens culturels - légitimes ou non - sur la recherche de contre-stéréotypes de genre. Autre reflet des caractéristiques des enquêteurs et enquêtrices, les entretiens recueillis témoignent de pratiques de lecture dignes de moyens voire de forts lecteurs, y compris chez certains garçons, par conséquent bien au-dessus de la moyenne des grands lecteurs adolescents et jeunes adultes estimés à 15 \% par l'enquête 2008 des Pratiques culturelles des Français. Dernier effet remarquable lié à la proximité par réseau entre les étudiant.e.s et leurs enquêté.e.s, les lycéennes interrogées sont particulièrement lectrices de littérature young adult (voir le second encadré). 


\section{Les « scripts » amoureux au prisme des biens culturels}

\section{La reconduction des stéréotypes de la romance : des hommes qu'il faut changer}

Dans le schéma narratif typique de la romance tel que l'a analysé Janice Radway au prisme des rapports de genre, l'homme est peu attentionné envers sa partenaire, distant, froid, voire brutal et violent - elle souligne à ce sujet que certaines scènes de premières relations sexuelles entre le héros et l'héroïne sont tout bonnement des viols. À l'issue du roman, l'homme a été transformé en mari doux et compréhensif. Ce schéma narratif d'un homme qu'il faut changer, changement dont la responsabilité pèse sur l'héroöne, se retrouve de prime abord dans les appropriations que nous avons mises en évidence. Car si les lycéen.ne.s notent que l'amour fait souffrir, et font souvent mention de la métaphore des aiguilles qui déchirent le cœur de Jack dans le livre (adapté aussi en film d'animation) Jack et la mécanique du cœeur, il faut noter que Jack est une exception masculine qui contredit l'image de «la phobie de l'engagement » masculine popularisée par la presse psychologique et analysée sous un angle sociologique par Eva Illouz (2012). C'est ce qui ressort de l'entretien de Jessica (17 ans, père imprimeur, mère esthéticienne, $1^{\text {ère }} S$ ), lorsqu'elle me relate l'histoire de Jack :

«C'est une dame qui trouve un bébé et qui doit lui fabriquer un cœur et il n'a pas le droit de sortir, il désobéit et tombe amoureux sauf que c'est très dangereux pour lui et vu qu'il s'enfuit sa mère adoptive meurt de chagrin, après je me rappelle plus de l'histoire. [...] La fin est triste vu que le garçon meurt quand même, mais ça m'a plu ce qu'il fait pour retrouver la fille ou sa mère [..] Parce que ce que m'ont répété mes amis c'est que dès qu'il y a un danger ou un effort à faire, y a des gens qui s'arrêtent vite fait on va dire. »

6 Ces représentations "négatives" de l'amour ne sont pas généralisables, puisqu'à l'inverse Ulysse (17 ans, fils de viticulteurs, $1^{\text {ère }}$ S) mentionne comme histoire d'amour qui l'a marqué le film Eternal sunshine of the spotless mind : «ça m'a rappelé combien ça peut être beau et doux parce que j'ai connu ça une fois ». Lisiane (mère au foyer, père cadre bancaire, Terminale S), qui à 18 ans est en couple depuis quatre ans et demi avec un garçon qui fait partie intégrante de sa famille, ne regarde que des films d'amour «qui finissent bien» et évite les autres. Les biens culturels sont donc retenus, au double sens d'être pré-sélectionnés et d'être mémorisés, lorsqu'ils viennent conforter des croyances intériorisées à ce moment de la trajectoire.

Outre les scripts classiques de l'amour qui fait souffrir, on peut noter que ce sont généralement les hommes qui sont responsables de cette souffrance dans certaines représentations des lycéens, en écho au prisme des schémas narratifs de la romance.

8 Ainsi Lucas (18 ans, mère au chômage, Terminale ES) parle-t-il du réalisme du film L'Arnacoeur, ce dernier mettant en scène un personnage de Don Juan :

«Y a un film qui me vient mais c'est pas pour tous les garçons. C'est l'Arnacoeur, c'est un type qui passe son temps à traîner avec des filles et puis un jour il va tomber sur LA fille et il va se rendre compte qu'il se comportait pas comme il faut, et puis même là il se comporte pas comme il faut et au final ça se passe bien. C'est le but des films.

- Pourquoi ça vous semble réaliste?

- Parce qu'il y en a beaucoup qui agissent comme ça et à tout âge. Ma mère sort en boîte tous les vendredi et samedi soir, bon elle a 43 ans, elle est pas non plus hyper âgée mais elle tombe sur beaucoup de mecs en boîte qui ont un comportement... 
qu'ils aient 30 ans, 50 ans, 60 ans, du moment qu'ils sont en boîte ils redeviennent des animaux. Moi aussi je suis déjà allé en boîte, et c'est pareil pour les gens de mon âge. C'est parce qu'il y en a plus qu'on ne le pense que je trouve que ce film est réaliste.»

9 Cet extrait montre que le film vient conforter une croyance antérieure dans le comportement prédateur des hommes, croyance établie sur les récits d'expérience de la mère avec laquelle Lucas vit seul. Pourtant, le film se conclut par une évolution positive mais « c'est le but des films », ce qui laisse entendre qu'une telle évolution reste fictive.

Si Lucas confirme ses expériences antérieures par la fiction, Louisa (17 ans, mère auxiliaire de vie en France, père cuisinier au Maroc, $1^{\text {ère }} S$ ), pour sa part, s'inscrit dans un usage pratique d'apprentissage quelque peu désenchanté des livres et des films d'amour, qui lui permettent de vivre des expériences et d'en tirer des leçons par procuration. Elle ne peut pas parler de sa vie amoureuse avec sa famille et s'appuie sur les fictions littéraires ou cinématographiques pour mener sa vie amoureuse :

"Je vais à la bibliothèque et je prends. Il y a des romans d'ado, je cherche, je regarde un peu le résumé.

- Qu'est-ce que vous aimez bien dans ces romans d'amour?

- Ça fait apprendre des trucs, ça rend un peu plus forte par rapport à ma vie amoureuse à moi aussi. Des fois on apprend par les erreurs des autres. Des fois je tombe sur les mêmes erreurs et je sais comment réagir. [...] Par contre les chansons d'amour j'aime pas ça parce que j'aime pas pleurer. Les films d'amour y a $M a$ première fois, qui est un film d'amour que j'aime trop. C'est une fille qui avait 18 ans, elle a jamais eu de copain et la première fois qu'elle est tombée amoureuse c'est sa première fois, donc c'est toutes ses premières fois en même temps, et il la trompe. On apprend qu'il ne faut jamais faire confiance. [...] Il y a le livre Ma réputation. C'est une lycéenne de 16 ans, elle est sortie avec un garçon et il voulait prendre des photos sexy et vu qu'elle l'aime elle a accepté. Il l'a trompé donc elle a cassé et pour se venger il a montré les photos à tout le monde. La fille s'est même suicidée parce qu'elle en pouvait plus. Il a sali sa réputation. Et ça, ça nous apprend aussi à ne jamais faire confiance aux garçons. »

11 Dans cet extrait, on observe que les personnages féminins non seulement subissent de manière dramatique les conséquences des actes masculins, dont certains revêtent un caractère explicitement délictueux, mais encore que contrairement au schéma narratif de la romance, les femmes ne changent pas les hommes: elles en demeurent les victimes. Louisa en tire des leçons en se protégeant dans ses relations amoureuses hétérosexuelles. On peut remarquer que si le schéma narratif de la romance est globalement reconduit, on ne retrouve pas l'un des aspects, celui de l'action rédemptrice, pour les hommes et pour le couple à venir, des femmes. Les personnages féminins qui suscitent l'amour sont passifs, même le personnage masculin positif de Jack et la mécanique du cœur qui tombe amoureux est lui-même au centre du récit, et les scripts des relations amoureuses hétérosexuelles suscitent des identifications cathartiques tout en jouant un rôle préventif pour ce qui pourrait advenir dans la vie réelle. Ces premiers résultats obtenus lors de la phase d'enquête que j'ai menée dans deux lycées bordelais ont été nuancés dès lors qu'avec les deux classes d'étudiant.e.s, nous avons ciblé les personnages féminins et masculins marquants, en cherchant dans un second temps s'ils étaient reliés à un script amoureux. Vont alors émerger des identifications admiratives qui viennent contrebalancer les stéréotypes de genre de la romance. 


\section{Des identifications admiratives à des personnages contre- stéréotypés}

Si les identifications aux personnages qui ont été repérées dans les entretiens combinent les dimensions empathique, admirative et cathartique, l'admiration apparaît cependant fondamentale dans la majorité des cas. En effet, que les personnages trouvent un écho par ressemblance chez les lycéens et lycéennes ou qu'ils expérimentent des expériences extrêmes, ce sont leurs qualités jugées extraordinaires qui sont mises en exergue.

Il apparaît que les personnages féminins marquants sont caractérisés par leur autonomie et leur force morale, et ce, qu'il s'agisse de personnages de la littérature légitime comme Phèdre et Antigone, de la littérature young adult, des séries ou encore d'actrices et de femmes politiques réelles, alors que les personnages masculins, moins nombreux mais plus diversifiés, sont appréciés lorsqu'ils ne sont pas seulement forts mais aussi sensibles. De ce fait, les tableaux suivants rendent compte d'un clivage dans la perception des personnages selon leur genre: les personnages féminins sont nombreux à être cités par les filles et appartiennent majoritairement à la catégorie de la littérature young adult (voir encadré).

Tableau 1. Personnages féminins cités par les filles par catégorie de biens culturels

\begin{tabular}{|c|c|c|c|c|c|}
\hline $\begin{array}{l}\text { Type de bien } \\
\text { culturel }\end{array}$ & $\begin{array}{l}\text { Théâtre } \\
\text { classique }\end{array}$ & Young adult & $\begin{array}{l}\text { Bande } \\
\text { dessinée }\end{array}$ & Série & $\begin{array}{l}\text { Personnage } \\
\text { réel }\end{array}$ \\
\hline Personnage & $\begin{array}{l}\text { Antigone } \\
\text { Phèdre }\end{array}$ & $\begin{array}{l}\text { Margo, La Face cachée } \\
\text { de Margo } \\
\text { Hazel, Nos étoiles } \\
\text { contraires } \\
\text { Maddy, Everything, } \\
\text { Everything } \\
\text { Katniss, Hunger } \\
\text { Games } \\
\text { Tris, Divergente } \\
\text { Hermione, Harry } \\
\text { Potter } \\
\text { Ellana, Le Pacte des } \\
\text { Marchombres }\end{array}$ & $\begin{array}{l}\text { Maud, La } \\
\text { Rose écarlate }\end{array}$ & $\begin{array}{l}\text { Spencer Hasting } \\
\text { dans Pretty Little } \\
\text { liars } \\
\text { Jenna Hamilton } \\
\text { dans Awkward } \\
\text { Lydia dans Teen } \\
\text { Wolf }\end{array}$ & $\begin{array}{l}\text { Coco } \\
\text { Chanel } \\
\text { Simone } \\
\text { Veil }\end{array}$ \\
\hline
\end{tabular}

14 Les personnages féminins ci-dessus sont loués pour être « un personnage combatif et doté de force de caractère" (au sujet de Maud dans La Rose écarlate), des battantes (Margo, Hazel, Jenna Hamilton), ou encore « badass » (forte, indépendante, pour Lydia et Coco Chanel). Antigone est citée par une lycéenne «parce qu'Antigone lui a appris à dire non ». Enfin, un personnage apparaît à la fois sous son aspect fictif et son incarnation réelle: Hermione, jouée par Emma Watson, au sujet duquel Johanne déclare: " Elle incarne la beauté, l'intelligence, elle reste humble malgré sa célébrité, et mène un combat contre l'inégalité homme/femme. Elle est vraiment la perfection féminine ». 


\section{La catégorie éditoriale de la littérature " young adult "}

La littérature classée en young adult se caractérise par l'objectif de toucher un public-cible, les 14-20 ans, et non par une unité littéraire. Originaire des pays anglophones, cette littérature s'est développée à partir du titre Nos étoiles contraires de John Green qui relate une histoire d'amour entre deux adolescents atteints du cancer et qui relève de la sous-catégorie sick lit. La catégorie young adult recouvre partiellement plusieurs sous-catégories ou sous-genres : la bit lit, la new romance, les littératures de l'imaginaire, etc. Harry Potter est parfois considéré comme l'ouvrage fondateur de la young adult. Du côté des auteur.e.s français.es, on peut associer à cette catégorie les romans de Pierre Bottero ou encore de Clémentine Beauvais. Les éditeurs de young adult portent une attention particulière à la réception de leur lectorat, aux blogs et aux chaînes Booktube souvent tenus par des lectrices, voire même aux fan fictions rédigées par les adolescent.e.s.

La multiplicité des personnages féminins s'accompagne ainsi d'une uniformité de caractérisation : sont plébiscitées les femmes fortes, indépendantes, qui disent «non » comme Antigone, ou qui se déclarent féministes dans le cas d'Emma Watson. Ils sont exclusivement cités par des filles, ce qui correspond à la tendance déjà observée en sociologie de la réception selon laquelle les filles s'identifient à des personnages féminins comme à des personnages masculins (Détrez, 2011), ces derniers étant particulièrement valorisés dans les fictions, tandis que les garçons et les hommes s'identifient seulement à des personnages masculins et évitent d'être associés à des genres fictionnels ou des personnages féminins symboliquement dévalorisés (Albenga, 2007, 2011). La présence des livres, y compris scolaires, est écrasante. Cette tendance contraste avec les différents types de masculinité qui sont déclinées au travers d'un petit nombre de personnages masculins de biens culturels plus divers (films, mangas) dont rend compte le tableau ci-dessous :

Tableau 2. Personnages masculins cités par des filles et des garçons, selon la catégorie de biens culturels et le type de masculinité

\begin{tabular}{|l|l|l|l|}
\hline Type de masculinité/ Bien culturel & Manga & Série & Film \\
\hline Hommes forts & Dragon Ball z & & \\
\hline Hommes forts capables de pleurer & & & John Mc Clain, Piège de cristal, joué par Bruce V \\
\hline Hommes intelligents & & Tyron Lannister, Games of Thrones & Harry Potter \\
\hline Hommes sensibles, dévoués & & & Jack et la mécanique du cour \\
\hline Couples ou personnages gays & & Lito et Hernando, un couple de Sense8 & Les héros du film Le Secret de Brokeback Mountai \\
\hline
\end{tabular}

16 Si les hommes forts, y compris celui capable de pleurer, sont mentionnés par des garçons, c'est également le cas des couples gays de Sense8 et Le Secret de Brokeback Mountain. Filles et garçons apprécient les personnages masculins intelligents et/ou 
sensibles, et deux filles vont mentionner le mathématicien gay Alan Turing. À l'exception de Dragon Ball $\mathrm{Z}$ positivement jugé pour sa force physique, les personnages masculins, tout comme les féminins, sont appréciés en tant que contre-stéréotype par les filles et les garçons. Selon Éric Macé, «mis au centre du récit, le contre-stéréotype prend le contre-pied du stéréotype en proposant une monstration inversée » (2009). À ce titre, Laetitia Biscarrat (2016) a identifié quatre formes de rapports aux stéréotypes de genre dans les séries par de jeunes adultes: le déni des stéréotypes de genre; la légitimation des stéréotypes de genre; la reconnaissance implicite des inégalités de genre que sous-tendent les stéréotypes associés à la féminité. La quatrième et dernière posture de réception mise au jour témoigne d'une compréhension approfondie des stéréotypes de genre. Il s'agit selon l'auteure d'une «analyse pragmatique », car les enquêtés appréhendent les stéréotypes au regard de la relation entre la production, les contenus et les publics de ces fictions sérielles. Dans le cadre de notre enquête, c'est une posture hybride entre les deux dernières catégories qui apparaît: les lycéen.ne.s repèrent l'existence de stéréotypes et peuvent même considérer qu'ils font partie intégrante du jeu de la consommation culturelle, mais ils les critiquent non sous l'angle des inégalités, sinon sous l'angle des injonctions de genre qui pèsent sur les personnages féminins comme masculins. En témoigne l'exemple de Quentin (18 ans, père journaliste, mère aide de vie à domicile, $1^{\text {ère }}$ STMG). Quentin dit lire peu « environ dix livres par an, je pense ", et surtout ce qu'il est obligé de lire pour ses études ou de la science-fiction. Toutefois, il est un grand consommateur de mangas mais considère « les mangas, c'est comme les BD, c'est pas vraiment comme des livres pour moi, ça se lit plus vite et c'est moins prise de tête ». Si l'on analyse précisément les pratiques culturelles de Quentin, on peut remarquer que celui-ci n'est pas, contrairement à ce qu'il affirme, quelqu'un qui lit peu : à dix livres par an, il se classe parmi les moyens lecteurs selon les critères de l'enquête sur les Pratiques culturelles des Français (Donnat, 2009). Il est aussi un grand consommateur de séries et de films. Ses genres préférés sont les films d'action et les séries à enquêtes. Il remarque : «Tout le monde commence à en avoir assez de ces clichés, je comprends pas pourquoi les gens qui font ces films continuent à en mettre autant. Ça change pas la qualité du film, mais c'est mieux quand on a des personnages un peu différents de ceux qu'on a l'habitude de voir. » Il mentionne ainsi le film Piège de Cristal, dans lequel le héros, incarné par Bruce Willis, est un policier normal qui se retrouve en position de faiblesse, bien qu'il finisse par triompher de ses ennemis. "C'est le premier film d'action dans lequel j'ai vu le héros pleurer ». Le héros masculin qui pleure est un contre-stéréotype déjà identifié (Maigret, 1995).

17 Si les personnages masculins sensibles sont mentionnés par les filles comme par les garçons, les personnages féminins sont très majoritairement plébiscités par les filles, en particulier les personnages de la littérature young adult. Parmi les lycéennes qui ont mentionné la lecture de young adult, on trouve comme point commun qu'elles apprécient les caractéristiques des personnages féminins et font preuve de distance critique par rapport aux romances classiques. On peut ainsi citer Anaïs (17 ans, père est professeur des écoles, mère professeure des écoles à la retraite qui garde des enfants à domicile, Terminale ES) : dans les livres de young adult, "elle est attirée par des personnages féminins, car souvent ce sont elles les héros de young adult, qui est le genre qui l'attire le plus, personnages qui sont indépendants donc positifs. Selon elle, ce sont des modèles: "Ce sont des battantes, une vraie force de caractère, elles sont déterminées et se laissent dicter leur vie par personne ». Néanmoins, la force de 
caractère et l'autonomie des héroïnes s'inscrivent dans des scripts très majoritairement hétérosexuels, où leur dépendance affective à un personnage masculin vient limiter la portée subversive des contre-stéréotypes.

En outre, la young adult n'est pas exempte des stéréotypes concernant la place des personnages masculins dans la romance, comme le souligne Lucie, (16 ans, mère technicienne sur les péages, beau-père assureur, Bac Pro Commerce). Lucie lit des romans young adult et "amour adolescent ». Elle affirme ne s'identifier à aucun des personnages de ses lectures, surtout lorsqu'elle parle de young adult. Pour elle, « ce sont toujours des histoires d'amours qui finissent mal ». Elle raconte que le début de ces histoires est le même, "deux adolescents qui se rencontrent, on se sait pas trop comment et qui commencent à s'aimer ». Elle ne s'identifie pas à ces procédés, "c'est juste de la lecture ». Elle exprime un avis renseigné au sujet de la représentation stéréotypée dans les films du "mauvais garçon qui devient gentil », au sujet de laquelle elle trouve que "dans Ma première fois c'est plutôt bien représenté, à l'inverse de Titanic ». Au sujet de Ma Première fois, elle explique qu' « au départ c'est un voyou puis il évolue parce qu'il est amoureux d'elle et il fait tout pour elle alors qu'au début il était pas très sympa avec elle ».

On remarque que Lucie mobilise des compétences critiques pour comparer l'usage des stéréotypes dans Titanic et dans Ma première fois. Lucie est issue de classes moyennes peu détentrices de capital culturel et son type de baccalauréat ne la prête pas à développer des dispositions littéraires. Dans son cas, la lecture constitue une activité qu'elle ne peut partager avec son entourage, mais qui lui permet de développer des dispositions critiques: on voit que la pratique de la lecture a permis à Lucie de développer des dispositions critiques et réflexives, sur le fond du texte d'abord à l'égard duquel elle montre une distance - elle identifie les stéréotypes qui n'enlèvent rien à son plaisir de lecture (Houel, 1997) puis sur la forme du texte.

Ainsi, filles et garçons se rejoignent, dans leur réception des scripts amoureux, sur la perception négative du rôle des hommes dans les relations amoureuses comme je l'ai abordé dans un premier temps, et témoignent également d'une distance critique à l'égard des rôles assumés par chacun des deux sexes dans la romance. Ces résultats s'expliquent notamment par le fait que les lycéens et lycéennes qui sont en mesure de s'inspirer de biens culturels pour évoquer des scripts amoureux lors d'entretiens détiennent une forme de compétence réflexive et critique, qu'ils ont développé au fil de leur consommation de livres, de films ou de séries - souvent plusieurs de ces biens à la fois. La distance critique s'accompagne de la proposition d'un script romantique alternatif : celui du dévouement réciproque entre les deux partenaires d'un couple.

\section{Le script du dévouement réciproque : vers un horizon égalitaire?}

21 Le script amoureux qui recueille l'adhésion des lycéen.ne.s exprime un idéal selon lequel les deux sexes devraient faire preuve de dévouement dans les relations. Cet idéal semble aller dans le sens d'un élargissement de l'horizon d'attente amoureux vers une aspiration à l'égalité femmes-hommes dans les relations privées, du moins dans le domaine des représentations. En effet, le dévouement réciproque, souvent mis durement à l'épreuve, semble à l'asymétrie des rapports de genre du script de la romance - où les femmes travaillent à changer les hommes. En ce sens, il offre une 
perspective compatible avec l'indépendance et l'autonomie féminine plébiscitées à travers les personnages féminins cités par les lycéennes. Serait alors résorbée la tension contemporaine mise au jour par Eva Illouz (2012) entre désir d'autonomie et désir de lien affectif. Cet idéal s'accommode cependant de la norme du couple, ce en quoi il reconduit la binarité de genre et les engagements émotionnels très inégaux entre filles et garçons.

\section{Titanic, une réinterprétation du script de la romance}

Titanic (James Cameron, 1997) a en effet été mentionné dans 24 entretiens sur 58, contre 14 occurrences pour les films d'animation de Hayao Miyazaki et 9 pour Les Noces funèbres (Tim Burton, 2004). Ces mentions ont été à la fois spontanées et permises par la liste d'œuvres soumises aux lycéen.ne.s qui pouvaient choisir des œuvres qui leur avaient plu. L'entretien de Julie vient préciser les ressorts du succès de Titanic, tout en replaçant ses identifications aux personnages de fiction dans le processus de la mobilité sociale de sa famille.

Julie, 16 ans, en $1^{\text {ère }}$ ES dans les Yvelines, vit dans un village aisé, son père est chauffeur de bus, sa mère est assistante sociale. Elle est issue d'une famille de classe populaire qui vit depuis quelques années dans un milieu de classe moyenne haute. Elle suit le feuilleton Plus belle la vie avec sa mère. La télévision a un rôle fédérateur dans sa famille : «le soir on mange dans le salon devant la télé comme ça on partage un truc tous les trois ». Son film préféré est Intouchable car » Omar Sy s'occupe complètement de l'handicapé et puis il part de rien et il arrive à s'intégrer ». Cette réception peut être reliée avec le travail d'assistante sociale de sa mère et son envie de devenir infirmière, et met également en évidence une trajectoire sociale ascendante qui est celle de sa famille. Julie préfère les œuvres qui sont ancrées dans la réalité, c'est aussi pour cela qu'elle écoute essentiellement du rap. Elle lit davantage que ses camarades et n'est pas familière des grandes sagas littéraires qu'elle pourrait partager avec eux (Harry Potter, Hunger Games etc.) car il s'agit de fiction. Bien qu'elle semble rejeter les œuvres de fiction elle a été très marquée par le film Titanic. Elle mentionne que le personnage joué par Léonardo DiCaprio, Jack, est entièrement dévoué à sa petite amie : "Léo il est entièrement dévoué à sa copine, il ne fait pas semblant il est amoureux et il fait tout pour elle ». Elle établit un lien avec sa propre expérience, décevante, à savoir un seul petit ami pendant un mois, qui «n'avait pas de sentiments pour elle». Dans Titanic, les personnages cherchent tous les deux «la liberté et l'amour ». Elle note que les femmes sont souvent soumises dans ce qu'elle voit à la télévision et apprécie donc d'autant plus Rose de Titanic.

Bien que cela ne soit pas mentionné par Julie, l'histoire d'amour de Titanic se base sur l'antagonisme de classe sociale entre Jack et Rose, symbolisé par leurs places respectives dans le Titanic qui permettent d'en faire découvrir l'organisation hiérarchique et l'économie symbolique. Cet obstacle narratif à l'amour se situe dans la lignée de Roméo et Juliette qui appartiennent à des clans adverses. La supériorité de classe est également l'une des raisons, si ce n'est la principale, pour lesquelles Rose n'a pas à accomplir tout le travail émotionnel de la plupart des héroïnes de romance : elle a déjà un fiancé attitré appartenant à sa classe, son projet n'est pas de contracter un mariage avec Jack, tout au contraire la spontanéité du sentiment réciproque est opposée au mariage de convenance. Julie, à ce titre, dit être attirée par l'accord 
spontané entre les deux protagonistes pour rechercher à la fois « la liberté et l'amour ». On trouve ici un élément original à l'égard de l'analyse de Janice Radway (1984) : un homme qui n'a pas besoin d'être changé car il assumerait de pouvoir être amoureux d'emblée. Dans la réception de Julie, la dimension de classe est effacée, pourtant Jack peut assumer d'autant mieux un sentiment amoureux (et un désir sexuel) qui va de pair avec une transgression sociale à son avantage. Autrement dit, le sentiment amoureux est vécu également sur le mode masculin du défi envers une jeune femme déjà engagée avec un homme d'une classe sociale favorisée. En outre, l'immédiateté du dévouement de Jack n'est pas unanimement saluée dans les réceptions des lycéens et lycéennes: plus haut dans le texte, Lucie estimait que le script du «mauvais garçon qui devient gentil » était plus finement déroulé dans Ma première fois que dans Titanic, ce qui montre une fois de plus que l'appropriation que Julie propose de Titanic apporte essentiellement des informations sur l'importance qu'elle, comme nombre d'autres enquêté.e.s, accorde au dévouement.

Dans le déroulement de ce script du dévouement, le dépassement des obstacles liés aux normes sociales de constitution des couples ne concerne plus seulement l'homogamie sociale : il fournit également un script pour les histoires d'amour homosexuelles.

\section{L'idéal du dévouement comme dépassement des obstacles sociaux : homosexualité et transgression des normes de genre}

Les couples homosexuels évoqués sont beaucoup moins nombreux que les couples hétérosexuels, qu'ils soient fictifs ou vécus par les lycéen.ne.s ou des personnes de leur entourage. Les couples de lesbiennes sont encore plus rarement mentionnés que les couples gays qui apparaissent dans la série Sense8 et dans le film Le Secret de Brokeback Mountain. Ces couples-là sont évoqués comme des relations amoureuses qui doivent surmonter des obstacles, et à ce titre l'homosexualité représente un obstacle narratif au bonheur amoureux analogue aux différences sociales et ethniques - mentionnées par exemple par une lycéenne, Louise (17 ans, parents cadre et professeur, 1ère S), au sujet de la dystopie Entre chiens et loups. Ainsi, Alexandre (16 ans, dont les deux parents sont au chômage, 1ère L) parle en ces termes des couples de Sense $8:$ "J'adore Lito et Hernando, ils sont drôles, et puis des fois ils sont heureux, et ça me rend heureux aussi, juste, d'être ensemble. Dans leur pays ils peuvent pas trop se montrer ensemble, juste parce qu'ils sont gays et ça doit être horrible, mais ils surmontent ça et ils arrivent à être heureux. Je trouve ça très beau, des fois j'ai pleuré même». Le dévouement réciproque est ici mis à l'épreuve par la transgression de la norme hétérosexuelle, mais demeure associé à l'idéal du couple formé d'un "noyau dur de deux personnes " comme l'explique Clara (17 ans, père cuisinier, mère salariée dans une assurance, Première L). Clara a une sœur aînée, avocate, qui a souvent subi des insultes pour son homosexualité, ce qui sensibilise Clara au «fait de devoir trouver sa place dans une société qui a du mal à accepter d'autres types de relations que celle de l'hétérosexualité. » À ce titre, elle met en regard le film La vie d'Adèle et la bande dessinée qui l'a inspiré, Le leu est une couleur chaude. Selon elle, « le film a rendu vulgaire le profond sentiment d'amour présent dans la BD ». Elle dit n'avoir pas trouvé d'autres livres qui rendent aussi bien compte, à ses yeux, du sentiment amoureux. Dans sa conception de l'amour, un couple peut être libre. Elle parle de «noyau dur avec les 
deux personnes formant le couple mais ils peuvent chacun de leur côté aller voir d'autres personnes. »

Bien que Clara soit celle qui remette en question le plus de normes - celle de l'hétérosexualité, mais aussi de l'exclusivité sexuelle, elle défend le duo amoureux. Ces dérogations aux normes de genre demeurent donc conformes à un idéal amoureux du couple. Tout comme les contre-stéréotypes, elles se déploient dans un cadre binaire et ne remettent donc pas totalement en question l'imaginaire de la matrice hétérosexuelle fondée sur la complémentarité de deux parties (Butler, 2005). On peut ici considérer que la binarité du couple, en concentrant dans un duo le dévouement sans faille, audelà de la question de l'exclusivité sexuelle, nécessite dès lors un travail émotionnel privé très important. Cet investissement semble d'une part difficile à concilier avec l'injonction à l'indépendance et l'autonomie, et d'autre part implique une socialisation émotionnelle qui pour l'instant, s'avère être davantage l'apanage des femmes et des filles. Et en effet, l'idéal de dévouement réciproque se heurte à l'asymétrie qu'expérimentent filles et garçons dans leur investissement émotionnel et pratique dans les relations amoureuses.

\section{Un travail émotionnel réparti de façon asymétrique entre filles et garçons}

Cette asymétrie apparaît sous la forme d'un paradoxe : si les filles se disent moquées et sont moquées par les garçons de leur entourage concernant leurs goûts pour la romance, plusieurs garçons reconnaissent qu'ils se confient plus volontiers à des filles pour avoir des conseils en matière de relations amoureuses. Ainsi, Renaud (18 ans, Terminale $S$, mère au foyer, père neurologue), à la question de savoir s'il y a des activités qu'il partage davantage avec les filles, évoque spontanément « les discussions sur les relations » car les "filles sont plus subtiles »; cela ne l'empêche pas de considérer avec son père, neurologue, et ses frères que les films d'amour que regarde leur mère, femme au foyer, sont un objet légitime de moqueries. Ulysse (17 ans, fils de viticulteurs, $1^{\text {ère }} \mathrm{S}$,) pense également qu'il est plus aisé de parler de sentiments avec des filles et de sexualité avec les garçons. Jessica (17 ans, père imprimeur, mère esthéticienne, $1^{\text {ère }}$ ), pour sa part, craindrait de parler de sentiments avec des garçons, notamment ses frères, dont elle redoute qu'ils la jugent trop sensible, »cucul, chochotte, cœur d'artichaut ».

Il semble en aller de même pour les pratiques culturelles, films et livres, traitant de l'amour : elles ont une valeur d'usage pour le travail sur ses émotions et à ce titre, les filles sont reconnues pour leur compétence tout en ne pouvant pas toujours afficher ou partager leurs goûts culturels. Louisa, qui est la seule à échanger des conseils avec des garçons parce qu'elle a davantage d'amis garçons et que ses amies filles ne partagent pas son goût pour la romance, reconnaît que dans cet échange, c'est elle qui détient la plus grande compétence :

«Quand vous échangez avec vos amis garçons, vous trouvez que c'est vraiment réciproque, ils vous donnent autant de conseils que vous en donnez?

- Oui mais je trouve que c'est moi qui en donne le plus, j'ai plus d'idées franchement, je sais pas pourquoi, franchement ma vie sentimentale y a rien d'exceptionnel mais je connais plus de trucs. J'ai des amis, j'ai pas vécu plus de trucs qu'eux, mais je connais des trucs mieux qu'eux. Grâce aux livres et aux films. » 
Dans le cas de Louisa, ce travail émotionnel ne va pas de pair avec une focalisation plus forte sur l'amour que d'autres lycéennes. Lorsque je lui demande quelles sont ses priorités pour l'avenir, elle déclare :

«Mes études, aider les autres, un bon travail, un bon salaire, peut-être avoir un mari des enfants, mais ce serait le dernier truc. Parce que c'est avec des études que tu peux avoir un bon mari, parce que la place de la femme pour moi c'est pas avoir un mari, rester à la maison. [...] Quand tu es amoureuse, que tu penses à un homme, tu penses plus à ta vie.»

31 La dernière phrase montre clairement que la "compétence émotionnelle » est aussi une compétence pour soi, y compris dans une perspective émancipatrice. En revanche, les garçons n'évoquent pas leurs sentiments entre eux tout simplement parce que cela met en cause leur identité de genre. Lucas (18 ans, mère au chômage, Terminale ES) est celui qui développe le plus longuement cet aspect, et exprime son indignation face aux expériences de réassignations de genre dont il a été témoin :

«Nous les garçons on n'aime pas parler de nos sentiments parce que ça nous donne l'air fragiles. [...] Entre garçons, on n'en parle pas, je m'en souviens pas en avoir parlé sauf avec mes amis vraiment très proches, mais ils ne sont que deux ou trois. En fait, pour nous, avoir des sentiments crée une fragilité et les autres vont pouvoir s'engouffrer dedans et te faire du mal. [...] Moi j'ai connu un garçon au collège qui avait l'air un peu efféminé et les autres 'oh le pédé ...' mais qu'est-ce que ça peut te faire, même s'il est pédé, si t'as pas envie de le connaître trace ta route mais laissele tranquille.»

Ces constats d'un clivage de genre autour des discussions amoureuses ont été vérifiés par les entretiens menés par les étudiant.e.s. En effet, 28 filles parlent de relations amoureuses avec des filles et 17 filles en parlent avec des garçons, alors que 6 garçons en parlent avec des filles et 5 garçons avec des garçons. Les discussions amoureuses engagent donc bien davantage les filles. Les sociabilités et les échanges se nourrissent parfois de pratiques qui sont pourtant difficiles à partager car dépréciées : les films et les livres d'amour, qui semblent avoir une valeur d'usage importante à défaut d'avoir une valeur d'échange.

Ce paradoxe est illustré et explicité par Hamza (16 ans, mère employée et père ouvrier, Première ES). Hamza déclare ne regarder principalement que des films d'action, de super héros, et écouter des textes de rap. Pour lui ces consommations ne traitent pas d'amour, les personnages qu'il aime n'ont rien à voir avec l'amour, un sujet qui "n'intéresserait que les filles ». Pourtant, dans la lignée des résultats précédemment avancés, Hamza a mentionné le film Titanic dans les œuvres marquantes. Il repère également la présence de stéréotypes de genre et de contre-stéréotypes dans ses consommations culturelles: « souvent les hommes sont représentés comme plus forts, mais pour casser un peu cette image, il y a des super héros féminins qui apparaissent ». Plus intéressant encore, Hamza estime que les films sentimentaux «se basent toujours sur un personnage central féminin». On peut mettre en relation ce constat d'une défaillance de supports identificatoires masculins dans les fictions sentimentales avec le manque d'échanges masculins dans son entourage : il ne parle pas d'amour avec ses amis garçons, il en parle avec ses cousines lorsqu'un problème se présente avec sa petite amie, mais il souligne que ses cousines vont le conseiller de leur point de vue de femme. On perçoit ici combien pourrait importer le travail des créateurs et créatrices de fiction sur des personnages masculins identifiables sur le sujet de l'amour, peut-être 
par l'hybridation des genres artistiques entre des styles sentimentaux et des registres qui sont habituellement revendiqués par les garçons adolescents.

\section{Conclusion}

Cette enquête a permis de mettre en évidence plusieurs résultats consolidés par le nombre relativement important d'entretiens semi-directifs réalisés, en dépit et parfois grâce aux biais de la population enquêtée, composée principalement de filles des classes moyennes et notamment de lectrices de young adult. Le premier résultat a trait aux scripts amoureux que les lycéen.ne.s développent avec le support des biens culturels : ces scripts rendent compte d'une croyance répandue selon laquelle les hommes sont peu dignes de confiance en matière amoureuse et sont générateurs de souffrance dans l'expérience amoureuse. Ils correspondent à ce titre aux schémas narratifs de la romance mis en évidence par Janice Radway. Un élément un peu différent émerge : celui d'un horizon d'attente centré sur le dévouement réciproque au sein d'un couple hétérosexuel, les femmes gagnant en autonomie et en indépendance, les hommes en sensibilité. L'idéal mis au jour est donc celui d'un couple hétérosexuel sur la base duquel les assignations de genre seraient assouplies, ce qui ne remet pas en cause le système de genre ni la norme du duo conjugal.

On peut avancer, dans la lignée d'Eva Illouz, qu'un tel idéal permet de résoudre les tensions entre les aspirations à l'autonomie et celles qui tendent vers une réduction de l'incertitude affective : même en distinguant couple et sexualité, les lycéen.ne.s sont attachée.es à l'idée d'un engagement affectif réciproque qui loin de compromettre l'indépendance de chacun, semble offrir une assise plus forte à celle-ci puisque les deux membres du couple peuvent se soutenir mutuellement. Cet idéal se heurte cependant à une asymétrie de «travail émotionnel » entre filles et garçons liée à une socialisation de genre que les biens culturels renforcent en donnant la centralité aux personnages féminins dans les histoires d'amour. Pourtant, filles et garçons expriment leur souhait de lire des histoires moins stéréotypées, et si les personnages féminins forts et indépendants qui se multiplient semblent constituer un nouveau standard qui s'adresse au lectorat-cible que sont les filles adolescentes, demeure en suspens le développement de personnages masculins marquants pour leurs qualités sentimentales.

\section{BIBLIOGRAPHIE}

ALBENGA Viviane (2007), « Le genre de 'la distinction' : la construction réciproque du genre, de la classe et de la légitimité littéraire dans les pratiques collectives de lecture », Sociétés \&

Représentations, $\mathrm{n}^{\circ}$ 4(2), p. 161-176.

ALBENGA Viviane (2011), «Stabiliser ou subvertir le genre ? Les effets performatifs de la lecture », Sociologie de l'Art, OPuS 17, p. 31-43. 
BISCARRAT Laetitia (2015), « Le genre de la réception », Communication [En ligne], vol. 33/2, mis en ligne le 27 janvier 2016, consulté le 16 juillet 2018. URL : http://journals.openedition.org/ communication/5775.

BAudelot Christian, Marie CARTIER et Christine Detrez (1999), Et pourtant ils lisent, Paris, Seuil.

Bozon Michel (2016), Pratique de l'amour. Le plaisir et l'inquiétude, Paris, Payot.

BUTLER Judith (2005), Trouble dans le genre. Le féminisme et la subversion de l'identité, Paris, La Découverte.

CLAIR Isabelle (2007), « La division genrée de l'expérience amoureuse. Enquête dans des cités d'habitat social », Sociétés \& Représentations n² 24, p. 145-160.

CLAIR Isabelle (2011), « La découverte de l'ennui conjugal. Les manifestations contrariées de l'idéal conjugal et de l'ethos égalitaire dans la vie quotidienne de jeunes de milieux populaires », Sociétés contemporaines, $\mathrm{n}^{\circ}$ 83, p. 59-81.

CLAIR Isabelle (2016), « La sexualité dans la relation d'enquête. Décryptage d'un tabou méthodologique ", Revue française de sociologie, vol. 57, p. 45-70.

DETREz Christine (2011), « Des shonens pour les garçons, des shojos pour les filles ? Apprendre son genre en lisant des mangas », Réseaux, n 168-169, p. 165-186.

DonNat Olivier (2009), Les Pratiques culturelles des Français à l'ère du numérique, Paris, La Découverte/Ministère de la Culture et de la Communication.

GAGNON John H., ([1991] 2008), « L'utilisation explicite et implicite de la perspective des scripts dans les recherches sur la sexualité » dans J. H. GAGNON, Les scripts de la sexualité. Essais sur les origines culturelles du désir, Paris, Payot, p. 69-136.

HochschILD Arlie R. (2017), Le Prix des sentiments, La Découverte.

HouEL Annik (1997), Le Roman d'amour et sa lectrice. Une si longue passion. L'exemple Harlequin, Paris, L'Harmattan.

ILlouz Eva (2006), Les Sentiments du capitalisme, Paris, Seuil.

ILlouz Eva (2012), Pourquoi l'amour fait mal, Seuil.

JAUSS Hans Robert (1978), Pour une esthétique de la réception, Paris, Gallimard.

MACÉ Éric (2007), « Des 'minorités visibles' aux néostéréotypes », Journal des anthropologues, horssérie [En ligne]. http://jda.revues.org/2967. Page consultée le 20 août 2018.

MAIGRET Éric (1995), «'Strange grandit avec moi'. Sentimentalité et masculinité chez les lecteurs de bandes dessinées de super-héros ", Réseaux, volume 13, n 70, p. 79-103.

PASQUIER Dominique (1999), La Culture des sentiments. L'expérience télévisuelle des adolescents, Paris, Éditions de la Maison des Sciences de l'Homme.

RADWAY Janice A. (([1984] 1991), Reading the Romance. Women, Patriarchy and Popular Literature, Chapel Hill, The University of North Carolina Press, 1991 (1re éd. 1984).

\section{Bibliographie des œuvres citées}

\section{Livres}

La Mécanique du cœur, livre (Mathias Malzieu, Flammarion, 2007) ou Jack et la mécanique du cœur, album jeunesse (Mathias Malzieu, Flammarion, 2014) 
Harry Potter (J. K. Rowling, Folio Junior, 1997-2007)

Ma Réputation (Gaël Aymon, Actes Sud Junior, 2014)

La Face cachée de Margo (John Green, Gallimard, 2014)

Nos étoiles contraires (John Green, Pocket jeunesse, 2013)

Everything, Everything, (Nicola Yon, Bayard Jeunesse, 2015)

Hunger Games (Suzanne Collins, Pocket jeunesse, 2008-2010)

Divergente (Veronica Roth, Nathan, 2011-2014)

Le Pacte des Marchombres (Pierre Bottero, Rageot Editeur, 2006-2008)

Bandes dessinées

La Rose écarlate (Patricia Lyfoung, Delcourt, 2005-)

Le bleu est une couleur chaude (Julie Maroh, Glénat, 2010)

Films

Titanic (James Cameron, 1997)

Les Noces funèbres (Tim Burton, 2004)

Eternal sunshine of the spotless mind (Michel Gondry, 2004)

Le Journal de Bridget Jones (Sharon Maguire, 2001)

L'Arnacoeur (Pascal Chaumeil, 2010)

Ma Première fois (Marie-Castille Mention-Schaar, 2012)

Piège de cristal (John McTiernan, 1988)

Intouchables (Eric Toledano, Olivier Nakache, 2011)

Le Secret de Brokeback Mountain (Ang Lee, 2005)

La Vie d'Adèle - Chapitres 1 et 2 (Abdellatif Kechiche, 2012)

\section{Séries}

Game of Thrones (HBO, 2011-2019)

Sense8 (Netflix, 2015-2018)

Pretty Little liars (ABC Family, 2010-2015, Freeform 2016-2017)

Awkward (MTV, 2011-)

Teen Wolf(MTV, 2011-2017)

\section{RÉSUMÉS}

À partir d'une enquête réalisée par entretien auprès de 77 lycéens et lycéennes, cet article montre que les appropriations des films, livres, séries reconduisent et reconfigurent les rapports de genre qui traversent les relations amoureuses. La première partie met en évidence la reconduction des stéréotypes relatifs au rôle négatif des hommes dans la romance, qui tient de 
l'identification cathartique, ainsi que la recherche de contre-stéréotypes de la part de lycéens et lycéennes qui consomment des biens culturels en quantité et manifestent une identification admirative envers des personnages féminins forts et indépendants et des personnages masculins sensibles. La seconde partie questionne la portée égalitaire du script amoureux alternatif de dévouement amoureux réciproque revendiqué par les filles et les garçons, en montrant d'une part qu'il s'accommode du désir d'autonomie féminine comme de transgressions à la norme de l'hétérosexualité sans pour autant remettre en cause le duo conjugal; et d'autre part que sa portée alternative est limitée par la persistance d'une prise en charge plus grande par les filles du travail émotionnel.

Drawing on a fieldwork investigation led by interviews with 77 high-school students, this paper shows that the appropriations of movies, series and books by these students both reinforce and challenge gender relations at work in love relationships. The first part highlights how male characters still embody a negative role in the romance stereotypes that the students have incorporated and trigger a cathartic identification, whereas counter-stereotyped characters such as strong and independant female characters and sensitive male characters induce an admirative identification. The second part emphasizes an alternative love script that implies reciprocal devotion between the two partners of a couple, which is an ideal conveyed by both girls and boys. This ideal seems to comply with feminine autonomy and is not limited to heterosexual couples but do not challenge the binarity of the couple on which relies gender binarity. Moreover, its practical application is limited by the fact that girls still carry the load of emotional work much more than boys, so they are more likely to be devoted without reciprocity.

\section{INDEX}

Keywords : gender, love, reception, readings, movies, teenagers, emotional work

Mots-clés : genre, amour, réception, lectures, films, adolescents, travail émotionnel

\section{AUTEUR}

\section{VIVIANE ALBENGA}

Viviane Albenga est maîtresse de conférences en sociologie à l'IUT Bordeaux Montaigne, actuellement responsable du DUT Bibliothèques-Médiathèques-Patrimoine. Ses recherches portent sur les pratiques culturelles et artistiques saisies au prisme du genre, ainsi que sur les appropriations des idées féministes par la lecture et par les dispositifs d'action publique éducative. Sa thèse, récompensée par le prix de thèse de la Ville de Paris en études genre en 2010, a donné lieu à un ouvrage, S'émanciper par la lecture, Rennes, PUR, 2017. Elle travaille actuellement sur les appropriations pratiques du féminisme par la génération des étudiantes et les circulations de concepts féministes militants par les réseaux sociaux dans le contexte de l'après \#MeToo, en France et en Espagne. 